\title{
Escaping Particle fluxes in the atmospheres of close-in exoplanets: I. model of hydrogen
}

\author{
J. H. Guo ${ }^{1,2}$; guojh@ynao.ac.cn \\ National Astronomical Observatories/Yunnan Observatory, Chinese Academy of Sciences, \\ P.O. Box 110, Kunming 650011, China
}

Received —

\footnotetext{
${ }^{1}$ Key Laboratory for the Structure and Evolution of Celestial Objects, Chinese Academy of Sciences
} 


\begin{abstract}
A multi-fluid model for an atomic hydrogen-proton mixture in the upper atmosphere of extrosolar planet is presented when the continuity and momentum equations of each component have been already solved with an energy equation. The particle number density, the temperature distribution and the structure of velocity can be found by means of the model. We chose two special objects, HD 209458b and HD 189733b, as discussion samples and the conclusion is that their mass loss rates predicted by the model are in accordance with those of observation. The most important physical process in coupling each component is charge exchange which tightly couples atomic hydrogen with protons. Most of the hydrogen escaping from hot Jupiters is protons, especially in young starplanet system. We found that the single-fluid model can describe the escape of particles when the mass loss rate is higher than a few times $10^{9} \mathrm{~g} / \mathrm{s}$ while below $10^{9} \mathrm{~g} / \mathrm{s}$ the multi-fluid model is more suitable for it due to the decoupling of particles. We found that the predicted mass loss rates of HD $189733 \mathrm{~b}$ with the assumption of energy-limit are a factor of 10 larger than that calculated by our models due to the high ionization degree. For the ionized wind which is almost compose of protons, the assumption of energy-limit is no longer effective. We fitted the mass loss rates of the ionized wind as a function of $F_{U V}$ by calculating the variation of the mass loss rates with UV fluxes.
\end{abstract}

Subject headings: planets and satellites: atmosphere - planets: individual: HD 209458b, HD 189733b 


\section{Introduction}

The discovery that the hot Jupiter HD 209458b is losing mass was rather unexpected (Recently, Lecavelier des Etangs et al. (2010) have found atmospheric evaporation in HD 189733b. It is the second extrasolar planet whose mass loss has been detected.). The excess absorption in Lyman-alpha first found by Vidal-Madjar et al. (2003) (VM03) and later confirmed by Linsky et al. (2010) could be explained either by mass loss of the atmosphere due to XUV energy input from host stars (Lammer et al. 2003; Lecavelier des Etangs et al. 2004; Yelle 2004, 2006; Tian et al. 2005; Garcia Munoz 2007; Penz et al. 2008; Murray-clay et al. 2009; Lammer et al. 2009) or by charge exchange between the stellar wind and the planetary escaping exosphere (Holmström et al. 2008). For the former, all of these models describe the thermal particle escape. For the latter, Erkaev et al. (2005) and Holmström et al. (2008) brought forward loss of nonthermal neutral atoms due to interaction between the stellar wind and the exosphere (more details see Ekenbäck et al. 2010). The model of Erkaev et al. (2005) underestimates evidently the particle loss rates, but Ekenbäck et al. (2010) modeled the production of neutral hydrogen and match the feature of $L y \alpha$ absorption. It is not easy to distinguish which process dominates Lya absorption more. Ben-Jaffel \& Hosseini (2010) found that either energetic HI of stellar origin or thermal HI populations in the planetary atmosphere could fit Ly observations. Koskinen et al. (2010)(K10) used an empirical model to analyze UV transit depths, and their results showed that observations can be explained solely by absorption in the upper atmosphere while the

process of charge exchange could not be necessary. It is also noteworthy that the properties of planetary magnetic field are unclear until now. Thus, it is important for both thermal models and nonthermal models to calculate self-consistently the deflection distance around planet by using magnetohydrodynamics models.

The observations of transit in hot jupiters have found that the mass loss rates are in 
the range $10^{9}-10^{11} \mathrm{~g} / \mathrm{s}$ (VM03; Linsky et al. 2010; Lecavelier des Etangs et al. 2010). The effect of evaporation has been used to research the evolution of exoplanet. Baraffe et al. $(2004,2005)$ considered the effect of mass loss on the evolution of exoplanet, and mass loss rates varying from $10^{-12} M_{J} /$ year to $10^{-8} M_{J} /$ year are obtained from their calculations. However, an escape rate $10^{2}$ lower than Baraffe's $(2004,2005)$ is found by Hubbard et al. (2007a, 2007b). Their results showed that the moderate mass loss rates could be more appropriate for the mass function of observation. Guo (2010) further investigated the influence of mass loss on the tidal evolution of exoplanets, and found that the effect of mass loss on tidal evolution can not be neglect for those planets with initial mass $<1 M_{J}$ and initial orbital distance $<0.1 \mathrm{AU}$. It has also suggested that evaporation can lead to a significant modification to the nature of planet so as to form the planetary remnants (Lecavelier des Etangs et al. 2004, 2007; Penz \& Micela 2008; Penz et al. 2008; Davis \& Wheatley 2009).

At present, theoretical models focus on macroscopic material escape and the photochemistry is incorporated in their models (Yelle 2004; Tian et al. 2005; Garcia-Munoz 2007; Penz et al. 2008; Murray-Clay et al. 2009). All of these models suggested that the EUV and X-ray energy input by host stars is the main energy source for the escape of atmosphere. However, the escape process of different species may be dominated by different physical mechanism, and the winds around exoplanets orbiting their host stars at distant separation could be tenuous. Thus it must be argued whether the heavier species decouple from the light elements in tenuous winds (OI and CII transit depths of $13 \%$ and $7.5 \%$ obtained by Vidal-Madjar et al. (2004) indicated that both Oxygen and Carbon in the atmosphere of HD 209458b are lost.). Moreover, a detailed physical model is helpful probably to distinguish the origin of hydrogen around HD 209458b. Because much micro-physics has not included into hydrodynamic simulations, the study to the behaviors of species by using multi-fluid models may be worthwhile. 
With consideration of the process radiative transfer, this paper aims to calculate planetary atomic hydrogen and proton loss rates through the solution to their mass, momentum and energy equations. A great deal of microscopic physics processes are covered in the mass and momentum equations (Section 2.1). The code is designed for ordinary equations with one or more critical points (Section 2.2). We use Henyey method to calculate these equations (Section 2.4). The results are presented in Section 3. Of especial interest are what makes atomic Hydrogen and proton decoupling (Section 4.1)? In Section 4.2 we discuss the properties of ionized wind and fit the mass loss rate as a function of UV flux. We summarize my results in Section.5

\section{The model}

The model describes the steady state, radial expansion of plasma containing three species: atomic hydrogen (h), proton (p) and electron (e). Each species has its own continuity and momentum equations and is described by a particle density $n_{s}$ and velocity $u_{s}$. I do not include $H_{2}$ in this model because the thermosphere of close-in planet should be composed primarily of $\mathrm{H}$ and $\mathrm{H}^{+}$. The location of transition from $\mathrm{H}_{2}$ to $\mathrm{H}$ is about at $1.1 R_{P}$ (Yelle 2004). In addition, with the assumption of $T_{h}=T_{p}=T_{e}$, only one energy equation for electron is given in the calculations.

\subsection{Equations and assumptions}

As this model deals with a mixture of atomic hydrogen and proton, the following processes are considered: photoionization, recombination and charge exchange. The most important collision between neutral hydrogen and ion is charge exchange. Other collisions, as hard sphere collision, between neutral and ionized particle are not important. The 
continuity and momentum equations for each species including the source/sink terms which relate to photoionization/recombination and charge exchange for a species $j$ read as

$$
\begin{gathered}
\frac{1}{r^{2}} \frac{d\left(r^{2} n_{j} u_{j}\right)}{d r}=\frac{\delta n_{j}}{\delta r}(j=h, p) \\
u_{h} \frac{d u_{h}}{d r}+\frac{1}{m_{h} n_{h}} \frac{d P_{H}}{d r}+\frac{G M_{p}}{r^{2}}-\frac{3 G M_{*} r}{a^{3}}=\frac{\delta M_{s}^{h}}{\delta r} \\
u_{p} \frac{d u_{p}}{d r}+\frac{1}{m_{h} n_{p}} \frac{d P_{p}}{d r}-\frac{e E}{m_{H}}+\frac{G M_{p}}{r^{2}}-\frac{3 G M_{*} r}{a^{3}}=\frac{\delta M_{s}^{p}}{\delta r}
\end{gathered}
$$

where $\mathrm{G}$ is the gravitational constant, partial pressure $P_{j}=n_{j} k_{B} T$, temperature $T$, hydrogen atom mass $m_{H}$ and planetary mass $M_{p}$. The term $\frac{3 G M_{*} r}{a^{3}}$ denotes the tidal gravity. $M_{*}$ and $a$ are stellar mass and semi-major axis, respectively. $E$ is the charge separation electric field.

Quasi-neutrality and zero-current was assumed in the derivation of momentum equation, thus, $n_{e}=n_{p}$ and $u_{e}=u_{p}$. Both inertia and gravity are negligible in the electron momentum equation because of the small mass of electrons, so we can express the electric field $\mathrm{E}$ as

$$
e E=-\frac{1}{n_{e}} \frac{d P_{e}}{d r}
$$

The two terms: $\frac{\delta n_{j}}{\delta r}$ and $\frac{\delta M_{s}^{j}}{\delta r}(j=h, p)$, represent the source and sink terms caused by elastic and inelastic collisions with other species, respectively. The sources and sinks for the particle flux density are due to photoionization and recombination. The term of resonant charge exchange $H, H^{+} \longleftrightarrow H^{+}, H$ is included in the momentum equation but not in the continuity equation because before and after the interaction the same particles are present. With the assumptions and the definitions as above we write the collision terms as 


$$
\begin{gathered}
\frac{\delta n_{h}}{\delta r}=n_{p} \gamma_{r e c}-n_{h} \gamma_{p h o} \\
\frac{\delta n_{p}}{\delta r}=n_{h} \gamma_{p h o}-n_{p} \gamma_{r e c} \\
\frac{\delta M_{s}^{h}}{\delta r}=-\gamma_{r c e} n_{p}\left(u_{h}-u_{p}\right)-\gamma_{r e c} \frac{n_{p}}{n_{h}}\left(u_{h}-u_{p}\right) \\
\frac{\delta M_{s}^{p}}{\delta r}=-\gamma_{r c e} n_{h}\left(u_{p}-u_{h}\right)-\gamma_{p h o} \frac{n_{h}}{n_{p}}\left(u_{p}-u_{h}\right)
\end{gathered}
$$

where $\gamma_{p h o}=\frac{F_{U V} e^{-\tau}}{h \nu_{0}} \sigma_{\nu 0} s^{-1}$ and $\gamma_{r e c}=2.7 \times 10^{-13}\left(\frac{T}{10^{4}}\right)^{-0.9} n_{p} s^{-1}$ (Murray-Clay et al. 2009) represent the photoionization and recombination rates, respectively. $\gamma_{\text {rce }}=1.12 \times 10^{-8}\left(\frac{T}{10^{4}}\right)^{1 / 2}\left[1-0.12 \log \left(\frac{T}{10^{4}}\right)\right]^{2} \mathrm{~cm}^{3} \mathrm{~s}^{-1}$ is the rate of charge exchange (Geiss \& Burgi 1986).

We have assumed that ions and atoms have the same temperatures, which is justified by the high collision rates. For the proton, the temperature equilibration time in atomic hydrogen is

$$
t_{e q}=\frac{1}{n_{h} \gamma_{r c e}}
$$

Due to $n_{h} \sim 10^{5}-10^{11} \mathrm{~cm}^{-3}, T \sim 10^{4} \mathrm{~K}$, we have $t \sim 10^{-3}-10^{3} \mathrm{~s}$. Ionization time of about $10^{5} \mathrm{~s}$ (Yelle 2004) in the upper atmosphere is two orders of magnitude greater than the temperature equilibration time. Following Schunk (1975) the energy equation for electrons is given as

$$
\frac{3}{2} n_{e} u_{e} k_{B} \frac{d T}{d r}-k_{B} T u_{e} \frac{d n_{e}}{d r}=\sum_{j} \sqrt{2} Z_{j}^{2} \nu_{e e} m_{j}\left(u_{j}-u_{e}\right)^{2}+H-L
$$


The right terms of the equation denote in sequence elastic collisions with heavy ions, heating and cooling. As the assumptions of charge neutrality and zero-current the term of elastic collisions is zero. The heating process in the mixed flows is complex. The stellar radiation ionizes the species to produce high energy photoelectrons which share their energy with other species by collisions.

A fully description to the process is beyond the scope of this paper. I follow the model of Murray-Clay et al. (2009) to describe heating from photoionization, but a coefficient, $\alpha=\frac{n_{p}}{2 n_{p}+n_{h}}$, is used to denote the fraction which is shared with electrons. Therefore,

$$
H=\alpha \varepsilon F_{U V} e^{-\tau} \sigma_{\nu 0} n_{h}
$$

where $\varepsilon=\left(h \nu_{0}-13.6 \mathrm{eV}\right) / h \nu_{0}$ is the fraction of photon energy deposited as heat, $h \nu_{0}=20 \mathrm{eV}$, and

$$
\tau=\sigma_{\nu 0} \int_{r}^{\infty} n_{h} d r
$$

is the optical depth.

Murray-Clay et al. (2009) pointed out that the main contribution to cooling is Lya radiation. Although the radiation is emitted by the atoms and ions, the excitation of the atoms and ions is due to electron collisions. Thus, the process is an energy loss mechanism for the electrons. The cooling is

$$
L=7.5 \times 10^{-19} n_{h} n_{p} e^{-118348 / T} \operatorname{ergcm}^{-3} s^{-1} .
$$

\subsection{Critical points}

The equations considered here have the similar structure as the solar wind. From Eqs. (1)-(8) and (10), we find that there exist two singular (sonic) points where the velocity 
derivatives can not be determined by this set of equations. These singularities are important because the transonic solution is only one that can yield flows from subsonic velocity at the base of the wind to supersonic velocity at the outside of the singularity. At these points, the velocity derivatives must be obtained by additional conditions such as regularity conditions.

Eliminating all derivatives other than the velocity derivative from the $\mathrm{H}$ atom and proton momentum equations leads to a coupled differential equations of the momentum, which can be written in the matrix form

$$
\left(\begin{array}{cc}
a_{h h} & a_{h p} \\
a_{p h} & a_{p p}
\end{array}\right)\left(\begin{array}{c}
\frac{d u_{h}}{d r} \\
\frac{d u_{p}}{d r}
\end{array}\right)=\left(\begin{array}{c}
b_{h} \\
b_{p}
\end{array}\right)
$$

where

$$
\begin{gathered}
a_{h h}=\left(\frac{k_{B} T}{m_{H} u_{h}}-u_{h}\right) \\
a_{p p}=\left(\frac{10}{3} \frac{k_{B} T}{m_{H} u_{p}}-u_{p}\right),
\end{gathered}
$$

where vector $\mathbf{b}$ does not contain any of functions of the derivatives of the variables $n_{j}$, $u_{j}(\mathrm{j}=\mathrm{h}, \mathrm{p}), \mathrm{T}$.

In the case of the solar wind the locus of singularity can be defined by the points where the determinant of matrix $D=a_{h h} a_{p p}-a_{p h} a_{h p}$ vanishes (Burgi 1992). In this model we find $a_{p h}=0$, thus $a_{h h} * a_{p p}=0$ can be forced into $a_{h h}=0$ and $a_{p p}=0$. It means that there exist two separate critical points for atomic hydrogen and proton respectively, where their velocities equal their sound velocities. 


\subsection{Boundary conditions}

Six boundary conditions are necessary to solve equations (1)-(3), (10) and (12). At the bottom of the flow, we set $T_{0}=1000 \mathrm{~K}$. Here the particle densities are assumed to be $n_{h}+n_{p}=\rho_{0} / m_{H}$. According to the model of Murray-Clay et al. (2009) we assumed that

the value of density is $4 \times 10^{-13} \mathrm{~g} / \mathrm{cm}^{3}$. In the bottom regions the collisions between atomic hydrogen and proton are frequent, thus we assumed $u_{h}=u_{p}$. We need additional three boundary conditions to close the set of equations. Two boundary conditions are provided by the regularity condition. These conditions can be written as

$$
\frac{d H_{i}}{d r}=\sum_{j}\left(\frac{\partial H_{i}}{\partial y_{j}}\right)\left(\frac{\partial y_{j}}{\partial r}\right)=0 .
$$

$H_{i}$ is the momentum equation of species $i$, and $y_{j}$ is for variables $\mathrm{r}, \mathrm{T}, n_{k}, u_{k},(\mathrm{k}=\mathrm{h}, \mathrm{p})$. These boundary conditions are added to corresponding mesh points. However, in calculations we find that these conditions will result in numerically unstable. When the total number of mesh points is increased the approximate conditions $d u_{k} / d r=0(\mathrm{k}=\mathrm{h}, \mathrm{p})$ can be used successfully to substitute Equation (17)(Nobili \& Turolla 1988). For the last boundary conditions we assumed the optical depth $\tau_{c}^{h}=0.0023$ in the sonic points of atomic hydrogen, which implies that $\tau_{c}^{h}$ equals the optical depth between the sonic point and Roche lobe (Murray-Clayet et al. 2009).

\subsection{Numerical method}

The momentum equations have two singularities at the two points $u_{p}^{2}=\frac{10 / 3 \kappa T}{m_{H}}$ and $u_{h}^{2}=\frac{\kappa T}{m_{H}}$ (Eqs. 15 and 16) for proton and atomic hydrogen, respectively. The explicit form of equations (2) and (3) can be written as 


$$
y^{\prime}+\frac{g(x, y)}{f(x, y)}=0
$$

where $f(x, y)$ vanishes in the sonic points. Normally, the velocity of flow increases with radius. At a specific point (sonic point) $f(x, y)$ begins to change sign. Thus the critical points can be found at the location where the sign of $f(x, y)$ changes. To solving boundary problems, Henyey method is convenient and the critical points appearing within the integration domain can be treated consistently (Nobili \& Turolla 1988). As a modified Newton-Raphson method, the method includes the critical conditions into the set of linearized flow equations. All the variables $Y_{i}$ at half-mesh point are interpolated as

$$
Y_{x_{i+1 / 2}}=\frac{Y_{i+1}+Y_{i}}{2}
$$

while derivatives are approximated by finite differences

$$
Y_{x_{i+1 / 2}}^{\prime}=\frac{Y_{i+1}-Y_{i}}{x_{i+1}-x_{i}}
$$

In our calculations we allow for the specification of one condition every time a critical point is met.

\section{Results}

For the comparison with single-fluid model, firstly, The Murry-Clay et al. (2009) model is solved by their method and subsequently did again by Henyey method. No significant difference is found by different solving methods, while some minor differences could exist due to detailed numerical configuration. An advantage of using Henyey method is to obtain the solutions once at whole domains and to self-consistently handled the several critical points in numerical configuration. 


\subsection{HD 209458b}

In this section we applied our model to a typical and particular planet sample: HD 209458b with the radius of $1.4 R_{J}$, the mass of $0.7 M_{J}$ and the semi-major axis of 0.05 (Murray-Clay et al. 2009). The upper limit on the observed orbital eccentricity is 0.028(Ibgui \& Burrows 2009), so we assumed a circular orbit in the calculations. The mass of host star is $1 M_{\odot}$.

The results for HD 209458b are shown and the single-fluid results of Murray-Clay et al. (2009) are also plotted in Fig.1. Seen from Fig.1 clearly, except for a detailed difference, the multi-fluid model predicts much the same trends of temperature, velocity and particle number density as the single-fluid model does. The number density of atomic hydrogen decreases (upper left of Fig. 1) and the ionization fraction increases with radius (lower right of Fig. 1). The fact that as much as $80 \%$ of hydrogen at $R=5 R_{p}$ is ionized is a consequence of being irradiated by host star. With the increase of radius the optical depth becomes smaller, even near to zero, thus the radiation from star can freely penetrate the wind and ionize particles. Current studies show the wind temperature can attain 8000-10000K at about $2 R_{p}$ (Yelle 2004; Tian et al. 2005; Garcia Munoz 2007; Penz et al. 2008; Murray-Clay et al. 2009) and our results also verify the fact. The cooling by radiation and PdV work make the temperature reduced from $8000 \mathrm{~K}$ and decrease to about $5000 \mathrm{~K}$ in the upper atmosphere. The inversion of temperature in the upper atmosphere is a typical feature of planetary atmosphere. To compare our results with the results of other hydrodynamic models (Yelle 2004; Tian et al. 2005; Garcia Munoz 2007; Penz et al. 2008 and Murray-Clay et al. 2009), we found that all models obtain similar temperature profiles besides the model of Tian et al. (2005) which predicts a monotonously increasing temperature profile and a higher velocity structure. A possible reason is that Tian et al.(2005) applied a higher radiative heating. 
The transonic wind driven by stellar XUV has two sonic points: one is for atomic hydrogen and the other is for protons. Because protons bear an external electric force, E, with the assumption of the same temperature in both particles, the sonic point extends to outside of the wind. The velocity of $\mathrm{H}$ and $\mathrm{H}^{+}$is almost same. When one particle travels outward, the other particle drags it inward. The collision between two particles leads to a tight couple for their kinetics.

We have described the photoionization, recombination and charge exchange rates in Section 2.1. Evidently, charge exchange dominates the other two collision process $\left(n \gamma_{r c e} \gg \gamma_{p h o}, \gamma_{r e c}\right)$. To show the effect of charge exchange we calculate a model without charge exchange (In fact, we encounter numerical problems when the charge exchange is fully neglected. Thus we remain the term and multiply the term by a factor of $10^{-3}$.). Figure 2 displays that protons decouple from atomic hydrogen. If the most important process is neglected, the behavior of each particle looks like a single "fluid".

Note that the ratios of two velocity components decline with the increase of radius but they rise at a point again. The phenomenon can be explained by the behavior of photoionization and recombination rates. At the inner of the wind, the amount of momentum transferred by the process of photoionization and recombination is not enough, therefore neutral hydrogen decouple from protons. With the decrease of optical depth and temperature, the effect of photoionization and recombination rates on transferring momentum increases at the outer of the wind so that the value of $u_{h} / u_{p}$ also increases. A similar phenomenon will be discussed in Section 4.1.

The mass loss rates are defined as

$$
\dot{M}=4 \pi m_{H}\left(\left(r_{c}^{h}\right)^{2} n_{h} u_{h}+\left(r_{c}^{p}\right)^{2} n_{p} u_{p}\right)
$$



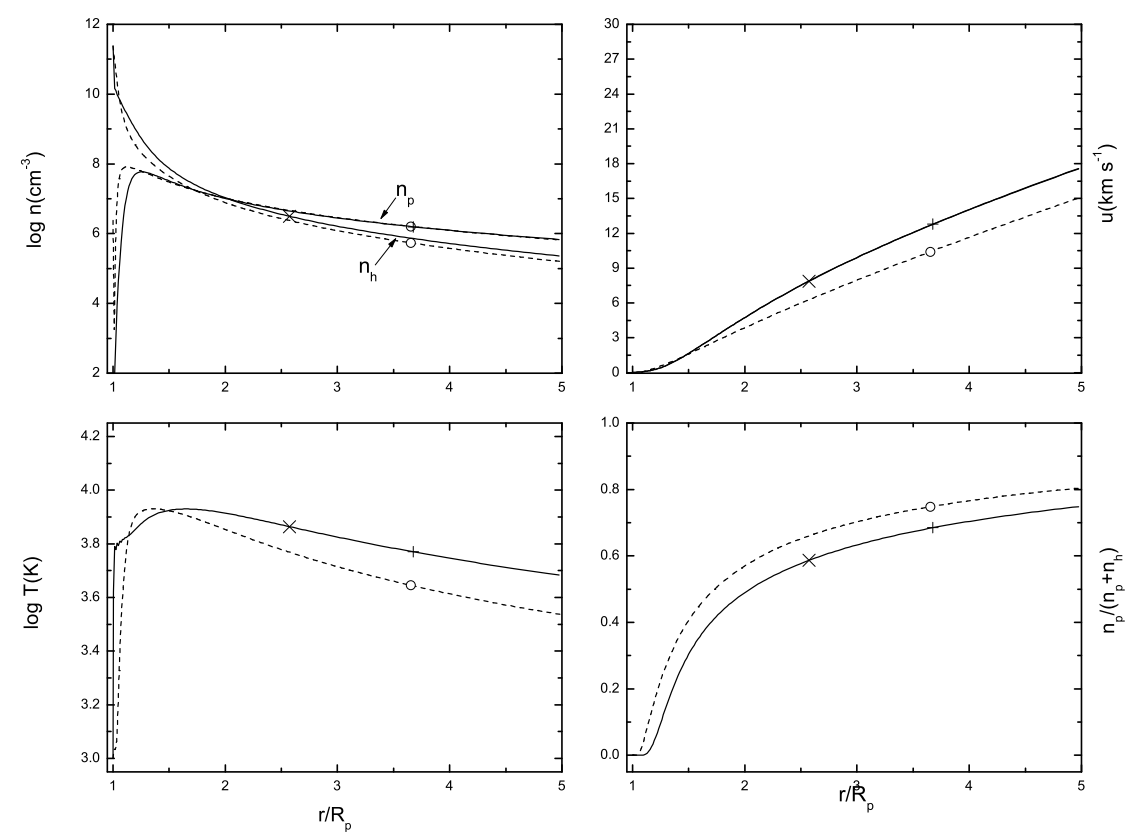

Fig. 1. - The wind model of HD 209458b. Dashed lines are the results of Murray-Clay et al. (2009). Solid lines represent our results. On each panel, the sonic point of single-fluid is marked with circles. The sonic points of $H$ and $H^{+}$are marked with $\times$and + , respectively. Number densities (upper left), velocities (upper right), temperatures (lower left) and the ionization fraction (lower right) are plotted as functions of altitude. Note that, for the number densities, the solutions of single-fluid and multi-fluid are indistinguishable. 


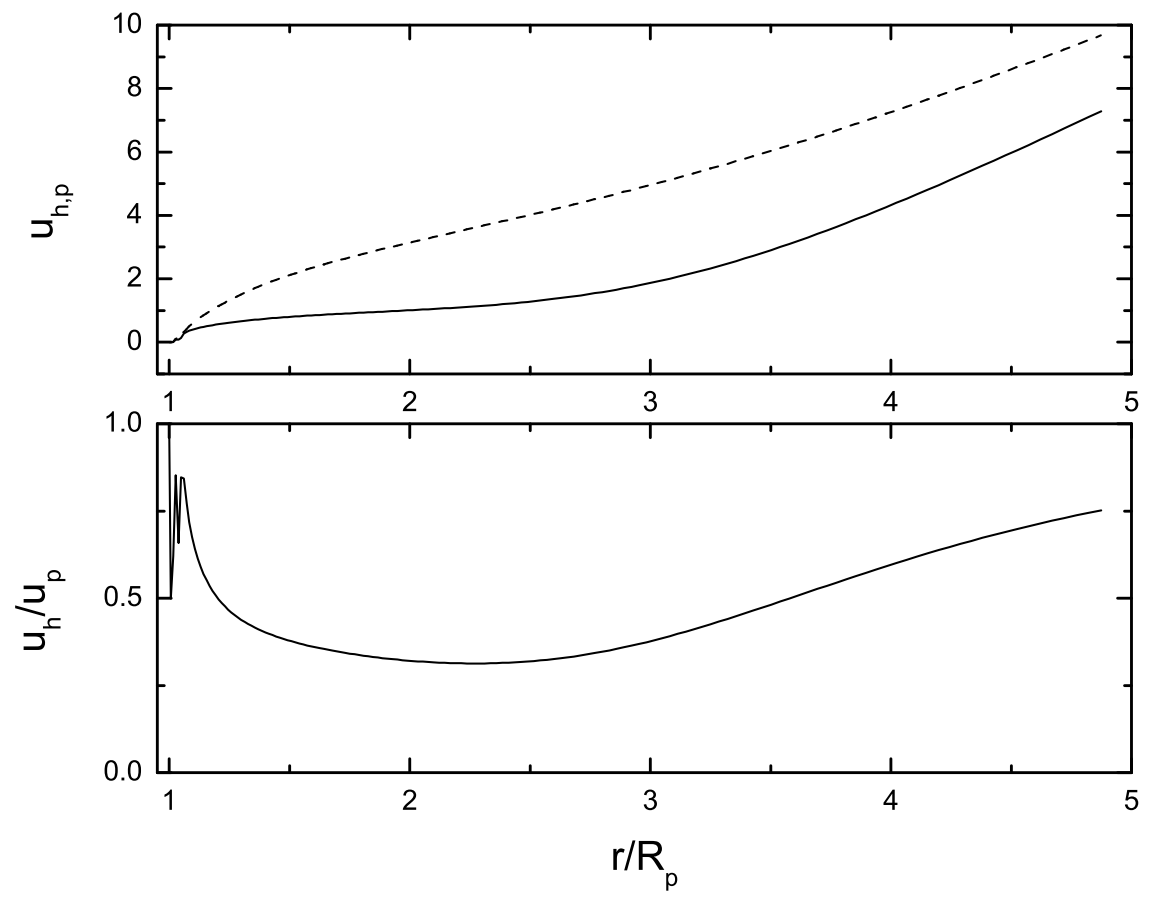

Fig. 2.- The effect of charge exchange on velocity profiles. The velocities of neutral hydrogen (solid line) and protons (dashed line) are indicated in upper panel. Lower panel shows the ratio of velocity, $u_{h} / u_{p}$, when the effect of charge exchange is neglected. 
where $r_{c}^{h}$ and $r_{c}^{p}$ are the sonic points of atomic hydrogen and protons respectively, $n$ and $u$ are corresponding number density and velocity. The mass loss rate of $\dot{M}=9 \times 10^{10}$ $\mathrm{g} / \mathrm{s}$ obtained by applying the solution over the entire planetary surface is higher than that of $\dot{M}=3 \times 10^{10} \mathrm{~g} / \mathrm{s}$ obtained by Murray-Clay et al. (2009), but both of them accord approximately with that of VM03 in observation. To fit the observed profile of $L y \alpha$, K10 assumed that the mean temperature of thermosphere is $T=8000-10000 \mathrm{~K}$ and the upper boundary is located at $R \sim 2.9 R_{p}$ where the number density is $n=2.6 \times 10^{7} \mathrm{~cm}^{-3}$. Our calculation results show that the particle number density at the radius $\left(R \sim 2.9 R_{p}\right)$ is $n=5 \times 10^{6} \mathrm{~cm}^{-3}$. A significant difference between their model and ours is that the assumption of hydrostatic equilibrium in their models. Our results show that the sonic points of $H$ and $H^{+}$are at $2.57 R_{p}$ and $3.67 R_{p}$, respectively. It means that the assumption of hydrostatic equilibrium is not unacceptable in the region range from $1 R_{p}$ to $2.57 R_{p}$. However, the number density distribution could be different due to the difference in physical detail. For example, our results that about $60 \%$ hydrogen is ionized at $2.9 R_{p}$ do not support the assumption of K10 that the atmosphere is mostly ionized above $2.9 R_{p}$. In addition, in the assumption of hydrostatic equilibrium the profile of number density is flatter than that of hydrodynamics, which can lead to high optic depth in the wings of line.

It is convenient to define the mass loss rates of neutral hydrogen and protons as

$$
\dot{M}_{h, p}=4 \pi m_{H}\left(\left(r_{c}^{h, p}\right)^{2} n_{h, p} u_{h, p}\right) .
$$

Our results indicate that the mass loss rates of neutral hydrogen and protons are $3.4 \times 10^{10} \mathrm{~g} / \mathrm{s}$ and $5.6 \times 10^{10} \mathrm{~g} / \mathrm{s}$, respectively.

To fit the observations of HD 209458b, two scenarios can supply a satisfactory fit. In the first case, thermal hydrogen atoms are enough to be used to fit the lyman alpha transit profile so that the energetic atoms are not necessary. In the second case, superthermal (hot) hydrogen atoms are required in order to fit the observations if depleted thermal 
hydrogen atoms are assumed (Ben-Jaffet et al. 2010). Superthermal hydrogen atoms in the atmosphere can be formed via the absorption of stellar UV radiation(Shematovich 2010). As hydrogen atoms have an excess of kinetic energy, they could be locally thermalized with the surrounding particles if the density of atmosphere is enough high. But, at the upper atmosphere, superthermal hydrogen atoms may escape from the atmosphere due to their excess of kinetic energy. A mass loss rate of $3.4 \times 10^{9} \mathrm{~g} / \mathrm{s}$ lower than the observational value for HD 209458b has been estimated by Shematovich (2010), and their results shown that those superthermal hydrogen atoms have velocities which are about $20 \mathrm{~km} \mathrm{~s}^{-1}$. Therefore, it is unlikely that superthmrmal hydrogen atoms can explain the observed velocities at the wing of line. Note that the location of transition from $H_{2}$ to $\mathrm{H}$ is about $1.1 R_{p}$ (Yelle 2004; also refer Shematovich 2010), this hints that there superthermal hydrogen atoms can not directly escape from the atmosphere of planet, but they must exchange energy and momentum with cool background particles in large-scale ranges. Exactly, the influence of superthremal hydrogen should be included into future hydrodynamic model.

\subsection{HD 189733b}

HD 189733b is the second extrasolar planet whose atmospheric evaporation has been detected (Lecavelier des Etangs et al. 2010). According to observations, they constrain the escape rate of atomic hydrogen to be between $10^{9}$ to $10^{11} \mathrm{~g} / \mathrm{s}$ and EUV flux is 10-40 times the solar value, namely, $F_{E U V} \simeq 1-4 \times 10^{4} \mathrm{erg} / \mathrm{cm}^{2} / \mathrm{s}$. The value may be changed to $2.5-10 \times 10^{4} \mathrm{erg} / \mathrm{cm}^{2} / \mathrm{s}$ if the emission of H I Ly $\alpha$ is also included. The observed value has been validated by X-ray observations. The luminosities of HD 209458 and HD 189733 in X-ray are measured as $\log L_{x}=26.12$ and $\log L_{x}=28.18 \mathrm{erg} / \mathrm{s}$ (Sanz-Forcada et al. 2010) by XMM-Newton. Because the orbital distance of HD 189733 is 1.5 times closer to its host star than that of HD 209458b, the planet receives X-ray radiation which is 300 times more 
than that of HD 209458b from host star. If assume that the flux in X-ray is proportional to UV flux, HD 189733b receives a UV flux of $F_{H D 19733 b}=F_{H D 209458 b} \times 300 \sim 10^{5} \mathrm{erg} / \mathrm{cm}^{-2} / \mathrm{s}$.

The planet has a mass $M_{p}=1.13 M_{J}$, radius $R_{p}=1.16 R_{J}$ and the semi-major axis $a=0.03 \mathrm{AU}$ (Bakos et al. 2006; Winn et al. 2007; Southworth 2010). The mass of its host star is $0.8 M_{\odot}$ (Nordström et al. 2004). Comparing with HD 209458b, the mass of HD189733b is 1.7 times larger than that of HD 209458b, and its radius is smaller. This means that the potential well of HD 189733b is about twice deeper than that of HD 209458b. Thus, the effect of strong X-ray flux can be balanced to a certain extent by its lager potential well.

Figure 3 displays the results for HD $189733 \mathrm{~b}$ with different values of $F_{U V}$. The mass loss rates are $4.8 \times 10^{10}, 1.1 \times 10^{11}$ and $1.98 \times 10^{11} \mathrm{~g} / \mathrm{s}$ for $F_{U V}=2 \times 10^{4}, 5 \times 10^{4}$ and $10^{5}$ $\mathrm{erg} / \mathrm{cm}^{2} / \mathrm{s}$. The mass loss rate is sensitive to UV flux received from its host star. Lecavelier des Etangs et al. (2010) found that the escape rate between $10^{9}$ to $10^{11} \mathrm{~g} / \mathrm{s}$ can fit Lyman $\alpha$ absorption of observation. However, we find that a value of $10^{9} \mathrm{~g} / \mathrm{s}$ can be obtained only when the UV flux decreases to $10^{3} \mathrm{erg} / \mathrm{cm}^{2} / \mathrm{s}$ which is one order of magnitude smaller than the lower limit of observed value ( $1 \sigma$ level). If the value of $F_{U V}$ is $10^{4} \mathrm{erg} / \mathrm{cm}^{2} / \mathrm{s}$, the mass loss rate of HD 189733 is $2.4 \times 10^{10} \mathrm{~g} / \mathrm{s}$.

In contrast to the results of HD 209458b, as much as $80 \%$ of hydrogen at $R=1.2 R_{p}$ is ionized and the wind is almost fully ionized outside $R=1.6 R_{p}$. The mass loss rate of neutral hydrogen is only of the order of magnitude of $10^{8} \mathrm{~g} / \mathrm{s}$ due to the photoionization of strong UV irradiation form host star (The number density of $H$ is of the order of magnitude of $10^{6} \mathrm{~cm}^{-3}$ at $R=1.6 R_{p}$ for the model of $F_{U V}=5 \times 10^{4} \mathrm{erg} / \mathrm{cm}^{2} / \mathrm{s}$. The corresponding temperature at the radius is $11000 \mathrm{k}$.$) . Actually, the wind is almost composed of protons.$ Under this circumstance, we wonder whether the neutral hydrogen can produce adequate absorption that can be detected (Due to the steep decline of the number density of neutral 

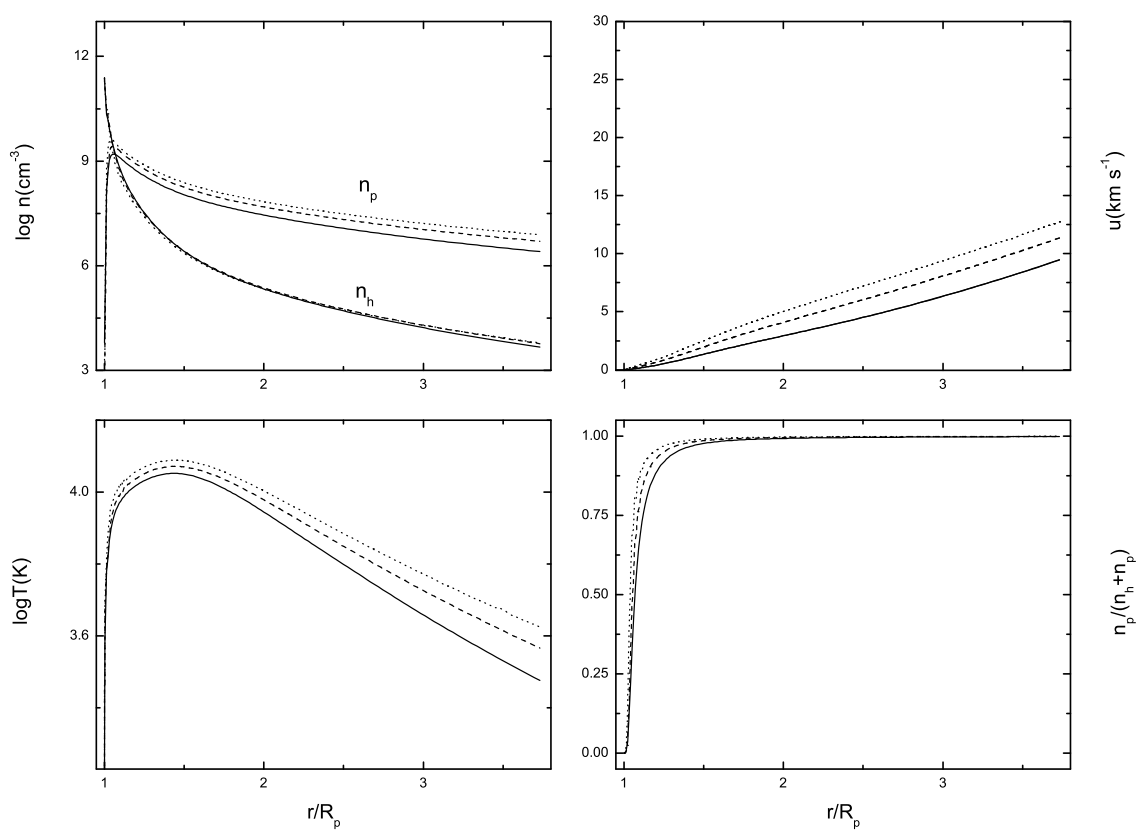

Fig. 3.- The wind model of HD 189733b. From top to bottom, the values of UV flux are $10^{5}$ (dotted lines), $5 \times 10^{4}$ (dashed lines) and $5 \times 10^{4} \mathrm{erg} / \mathrm{cm}^{2} / \mathrm{s}$ (solid lines). On each panel, number densities (upper left), velocities (upper right), temperatures (lower left) and the ionization fraction (lower right) are plotted as functions of altitude. 
hydrogen, the optical depth of wing of line can be very low.). If the amount of atomic hydrogen is not adequate to fit the observations, the fact that the transits of HD 189733b in HI Lyman- $\alpha$ have been observed may imply that others mechanisms can play an important role. A direct comparison with the model of Lecavelier et al. (2010) can answer the question. Unfortunately, the physical details of the model of Lecavelier et al.(2010) were not published.

\section{Discussions}

\subsection{Decoupling of species}

We have modeled the particle escape by a multi-fluid model. For the planet HD 209458b we do not find significant differences between the results of multi-fluid model with that of single-fluid model. In most cases the very close-in planets are bathed by strong XUV radiation from their host stars. The species of gas are tightly coupled by collisions so that the description of single-fluid is accurate. But, under certain special circumstances the description of single-fluid should be revisited or substituted, for example, when the planetary wind is tenuous or the irradiation from star is weak. For high number density, the frictional force is able to transfer sufficient momentum from one species to other species. However, decrease in the number density may lead to lowering in the frictional force, i.e. decoupling is possible.

The case is modeled by the method mentioned in this paper in order to discuss the possibility of the decoupling for $H$ and $H^{+}$. Figure 4 shows the ratios of two velocity components for HD 209458b and HD 189733b in their standard models. Neutral and ionized hydrogen have the same velocity over whole region. In order to test, HD 209458b and HD 189733b are moved to two times their original separations, namely, $a=0.1$ and $0.06 \mathrm{AU}$, 
and other parameters are retained. Seen from Figure 4, both the particles remain the same velocity profile. We also note that the number densities of particles only lower a factor of 2 than that of standard model.

The conclusion that even at large separation the description of single-fluid is still real seems to be drawn. However, it must be noticeable that the star HD 189733 is younger than HD 209458 (The ages of HD 189733 and HD 209458b are about 1.15Gyr (Sanz-Forcada et al. 2010) and 4Gyr (Guo 2010), respectively.), which means more XUV radiation can be emitted by HD 189733. If the age of HD 189733 is same as that of HD 209458, the conclusion may be different. For testing such a theoretical hypothesis, we calculated many cases with different values of $F_{U V}$ for HD 189733b. Seen from Figure 5, there exists a critical mass loss rate below which decoupling can occur (We assumed that decoupling would occur when $u_{h} / u_{p}$ is smaller than $95 \%$ in the calculations.). The results show that a significant diffusion between neutral hydrogen and protons occurs while the mass loss rate is below $1.3 \times 10^{9} \mathrm{~g} / \mathrm{s}$. Therefore the critical value is about $10^{9} \mathrm{~g} / \mathrm{s}$.

The conclusion is also verified by testing other cases. In order to decrease the mass loss rate of HD 209458b, we artificially increased the mass of the planet as $0.85 M_{p}$ and decreased the density of lower boundary by a factor of 10 . The results show that ionized hydrogen has a higher velocity profile and a clear decoupling occurs throughout the wind. Finally, we obtained a mass loss rate of $\dot{M}=5 \times 10^{8} \mathrm{~g} / \mathrm{s}$.

In order to obtain a common flow, the momentum gained by one species should be shared by other species. This requires that the characteristic time scale for slowing down the fast particle by interaction with the other slow particle should be smaller than the time scale of flow. The time scale of flow can be estimated as $R_{s} / u$, where $R_{s} \sim 0.1 R_{J}$ and $\mathrm{u}$ in $10^{5}-10^{6} \mathrm{~cm}$. The slowing down time scale, $t_{s}$, is approximately as $1 /\left(n_{h} \gamma_{r c e}\right)$. The condition of decoupling can be estimated by equaling two time scale. Therefore the critical 


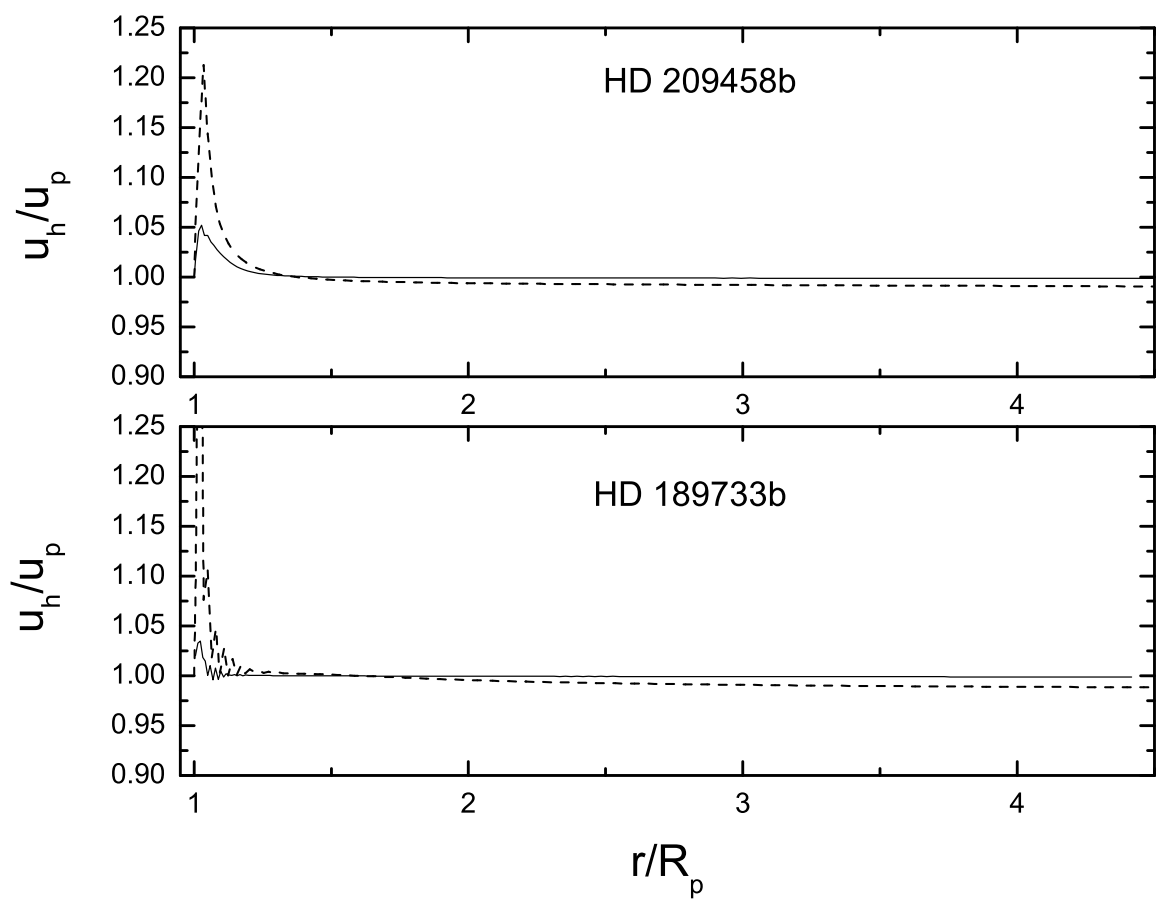

Fig. 4.- Plot of $u_{h} / u_{p}$ for HD 209458b and HD 189733b (dashed line: two times original separation; solid line: original separation.). In order to test the assumption of single-fluid, HD 209458b and HD 189733b are moved to two times their original separations. No significant diffusion was found in both cases. 


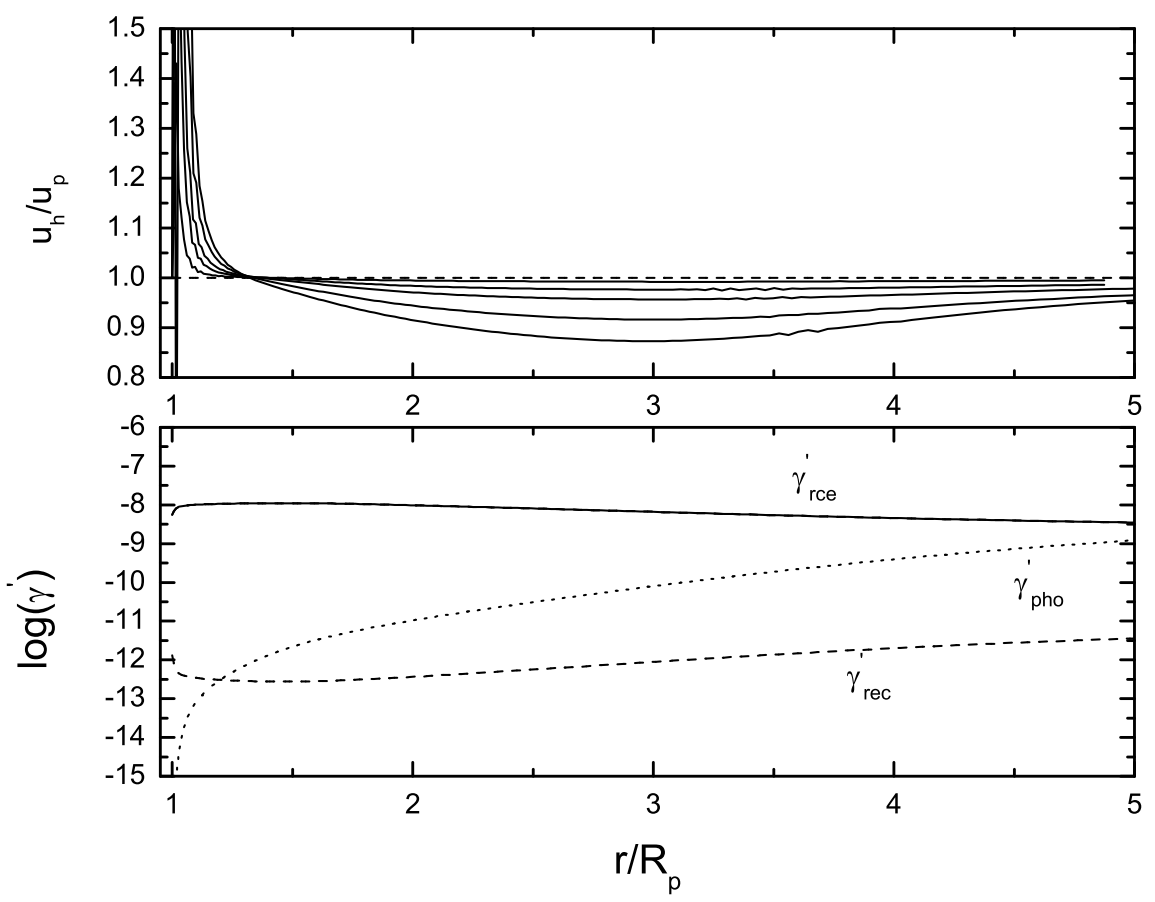

Fig. 5.- The velocity ratios of atomic hydrogen to protons (top panel) and the photoionization, recombination and charge exchange rates (bottom panel). In the top panel, the mass loss rates are $8.8 \times 10^{8}, 1.3 \times 10^{9}, 2.4 \times 10^{9} \mathrm{~g} / \mathrm{s}, 4.2 \times 10^{9}$ and $5.9 \times 10^{9}$. The corresponding UV fluxes are 450, 630, 1080, 1800 and $2500 \mathrm{erg} / \mathrm{cm}^{2} / \mathrm{s}$ (from bottom to top). The bottom panel only shows the photoionization, recombination and charge exchange rates in the model with $F_{U V}=450 \mathrm{erg} / \mathrm{cm}^{2} / \mathrm{s}$. For comparison, we defined these rates as: $\gamma_{p h o}^{\prime}=\gamma_{p h o} / n_{p}$, $\gamma_{r e c}^{\prime}=\gamma_{r e c} / n_{p}$ and $\gamma_{r c e}^{\prime}=\gamma_{r c e} \mathrm{~cm}^{3} s^{-1}$. 
number density of neutral hydrogen is about $10^{4}-10^{5} \mathrm{~cm}^{-3}$. Due to the $n_{p} / n_{h} \sim 10$, the critical mass loss rate is at the order of magnitude of $10^{9} \mathrm{~g} / \mathrm{s}$.

As mentioned in Section 3.1, the ratios of two velocity components behave as parabolic curves. To maintain the common flow, a sufficient amount of momentum must be transferred between two components. The most important process of transferring momentum is charge exchange. Near the bottom of the wind, the wind is relatively dense so that the process of momentum exchange is effective. However, with the increase of radius the rate of charge exchange decreases (The solid line at the bottom of Figure 5.). Thus, the transfer of momentum is also decrease with radius. We note that the process of photoionization and recombination can also redistribute momentum from one component to the other component, and the variations of photoionization and recombination rates (dashed and dotted lines) are contrary to that of charge exchange rate. Therefore, at the outer of the wind the momentum transfered by photoionization and recombination also plays a role. Finally, there is minimum value of $u_{h} / u_{p}$ in the middle of wind.

\subsection{Dependence of $\dot{M}$ on UV fluxes}

Lammer et al. (2003) presented that the energy deposition of X-ray and UV radiation from parent star can lead to a high temperature, and that a hydrodynamic process can occur in planetary atmosphere. The mass loss rates of energy deposition tightly depend on the fluxes of XUV radiation. In general young stars can radiate more energy than old ones in XUV band. The energy-limit mass loss rate can be written as

$$
\dot{M}=\frac{3 \eta \beta^{3} F_{s}}{G \rho K(\xi)}
$$

where $\beta$ is the ratio of the expansion radius $\mathrm{R} 1$ to the planetary radius $R_{p}, \mathrm{R} 1$ is altitude where the XUV radiation is absorbed, $R_{p}$ represents the distance form the center of planet 
to the 1 bar pressure level in the atmosphere, $\eta$ is heating efficiency, and $\rho$ is the mean density of planet. Here $K(\xi)=1-\frac{3}{2 \xi}+\frac{1}{2 \xi^{2}}<1$ is a non-liner potential energy reduction factor due to the stellar tidal force (Erkaev et al. 2007), and $\xi=d\left(\frac{4 \pi \rho}{9 M_{*}}\right)^{1 / 3}$ is Roche lobe boundary distance, where $M_{*}$ is the mass of star and $d$ is the orbital distance.

Based on the hydrodynamic model of Watason et al. (1981), Lammer et al. (2003) estimated $\beta=3$, but it could be unit according to recent hydrodynamic models which showed that the expansion radius could be $1-1.5 R_{p}$ (Yelle 2004; Murray-Clay et al. 2009). Lammers et al. (2009) also thought the mass loss rate was overestimated with $\beta=3$ by Barraffe et al. (2004). With the full energy-limited condition, the heating efficiency $\eta=100 \%$. In fact, the heating efficiency is about $25 \%$. In this paper, we set $\beta=1.1$ and heating efficiency $\eta=0.1-0.25$ (Murray-Clay et al. 2009 and refer therein). Thus, Equation (23) describes a modified energy-limit approach.

For comparison with the energy-limited mass loss rate, we calculated the mass loss rate of HD 189733b as a function of UV flux and the results is shown in Figure 6 (left panel). The mass loss rates given in our models are a factor of 3-10 lower than those calculated by Equation (23) at the assumption of $k(\xi)=1$ and $\eta=0.1-0.25$. For completeness we also calculated the single-fluid model (Murray-Clay et al. 2009) and found a systemic difference in comparing with the mass loss rates predicted by the model of this paper. For single-fluid model, it can predict a comparable value for $\dot{M}$ when the heating efficiency in the energy-limit method is decreased to $\eta=0.1$ (the left panel of Fig.6). However, this is not consistent with our results. The mass loss rates calculated by single-fluid model is still higher than these of our model. With the increase of $F_{U V}$, the ratios of $\dot{M}_{\text {single }} / \dot{M}_{\text {thispaper }}$ decrease from $5\left(F_{U V}=450\right)$ to $2.5\left(F_{U V}=10^{5}\right)$. As discussed in Section 3.2, our results fit the observations well. Thus, a lower heating efficiency is required for high ionized wind.

Our calculation results show that the winds are highly ionized and almost composed 
of protons even if the UV fluxes are assumed at a low level. The high ionization degree can be explained as the consequence of low mass loss rate. Even if the UV flux is at same level, the mass loss rate of HD 189733b should be lower than that of HD 209458b due to the larger potential well of HD 189733b. Thus, for HD 189733b, the low mass loss leads to the low optical depth and high ionization degree. Given the energy equation, it is clear that the photoionization heating is proportional to the number density of neutral hydrogen. With the assumption of energy-limit most of the UV radiation energy is deposited as heat (due to the low ionization degree), which is used to lift material out of the gravitational potential well. Thus, the condition of energy-limit results in higher mass loss rates. In the case of ionized wind the material is mainly composed of protons. Only a little of UV radiation can be transformed as heat, and further goes into PdV work. Murray-Clay et al. (2009) found that at high $F_{U V}$ the flow is radiation-recombination-limited (at low flux $\dot{M} \propto F_{U V}^{0.9}$; at high flux $\left.\dot{M} \propto F_{U V}^{0.6}\right)$, and an almost isothermal wind is predicted. In contrast to the case of radiation-recombination-limit, our results show a "normal" temperature profile. It hints that heating is balanced by PdV work rather than radiation cooling. Thus, we can summarize that the modified energy-limit approach can be used in the case of low or moderate ionization degree, but is unsuccessful for the high ionization winds. The conclusion can be validated by comparison with HD 209458b, we find that the mass loss rate calculated by Equation (23) can predict a reasonable observation value for HD 209458b.

The interesting results motivated us to fit the mass loss rate as a function of UV flux in the case of ionized wind. Seen from Figure 6, it is clear that the straight line can depict the lower part, and the upper part can be fitted by using a polynomial (a jump appears at $\left.F_{U V}=2000\right)$. Finally, the mass loss rate can be express as

$$
\dot{M} \bar{\rho}=\left\{\begin{array}{l}
-0.24+0.017 F_{U V}, F_{U V} \leqslant 2 \times 10^{4} \\
34.1+0.018 F_{U V}-3.92 \times 10^{-8} F_{U V}^{2}, 10^{5} \geqslant F_{U V}>2 \times 10^{4}
\end{array}\right.
$$



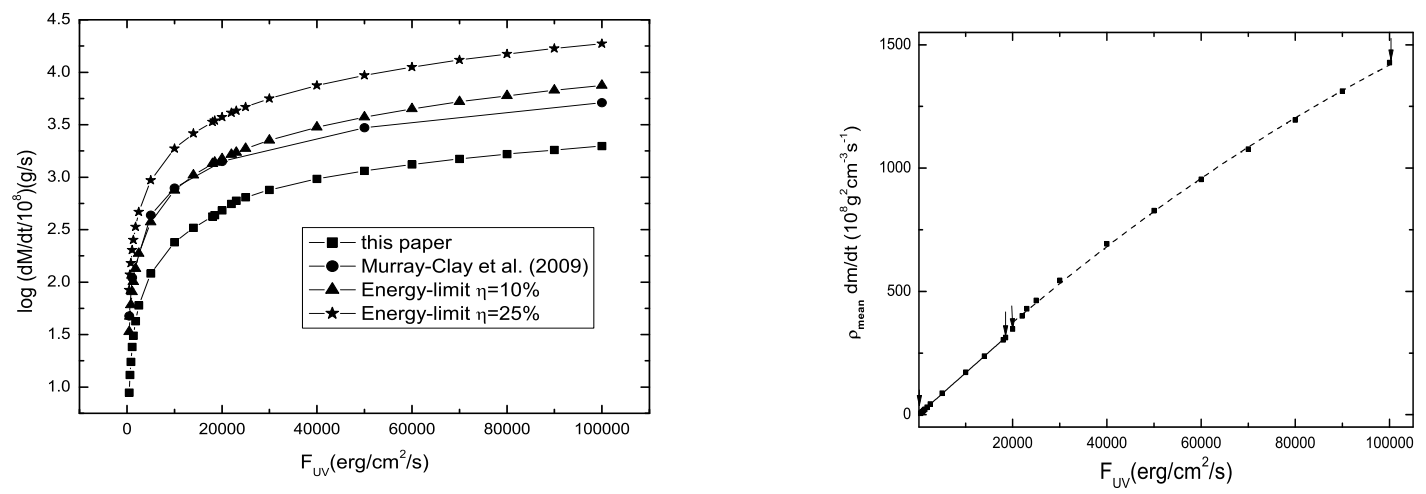

Fig. 6. - Left:The behaviors of mass loss rate as a function of UV fluxes. The results of energy-limit ( $\mathbf{\Delta}$ :heating efficiency $\eta=10 \%$; $\star$ : heating efficiency $\eta=25 \%$.) clearly show larger mass loss rates than those predicted by hydrodynamic models( $\bullet$ : the model of MurrayClay et al. 2009; $\mathbf{\square}$ : the model of this paper.). Right:The mass loss rates as a function of UV fluxes. For $F_{U V} \leqslant 2000 \mathrm{erg} / \mathrm{cm}^{2} / \mathrm{s}$, we used a liner-fitting (solid line). We fitted the rest of the data with a polynomial (dashed line). 
where $\bar{\rho}$ is the mean density of planet.

Note that Equation (24) is only appropriate for ionized planetary wind. But, it is not an easy task to determine whether the wind is ionized. To maintain an ionized wind, the photoionization rate should be larger than recombination rate, namely, $\gamma_{p h o}>\gamma_{r e c}$. Thus, we have

$$
\frac{F_{U V}}{h \nu_{0}} n_{h} e^{-\tau} \sigma_{\nu_{0}}>n_{p}^{2} \gamma_{r e c}
$$

At $R=1.5 R_{p}$, the optical depth $\tau \sim 0$, and $T \approx 10000 \mathrm{k}$. If the wind is ionized highly at $R=1.5 R_{p}$, the value of $n_{p} / n_{h}$ is about $10-100$. Inequality (25) can be changed to

$$
\frac{F_{U V}}{h \nu_{0}} \frac{\sigma_{\nu_{0}}}{\gamma_{r e c}}>\frac{n_{p}}{n_{h}} n_{p}
$$

With the assumption of $F_{U V}=10^{3} \mathrm{erg} / \mathrm{cm}^{2} / \mathrm{s}$, the left of inequality (26) is about in the order of magnitude $10^{9}$. Using the hydrostatic density profile, we can estimate the particle number density at $R=1.5 R_{p}$. For $\mathrm{HD} 209458 \mathrm{~b}, n_{p} \sim 7 \times 10^{9}$

so that $\frac{n_{p}}{n_{h}} n_{p}$ is of the order of $10^{11}$. Because the left term of inequality (26) is smaller than the right term, the wind of HD 209458b is not ionized. For HD 189733, $n_{p} \sim 10^{8}$. Thus the inequality (26) can be fulfilled roughly if $F_{U V} \geqslant 10^{3} \mathrm{erg} / \mathrm{s}$.

\subsection{The effect of magnetic field}

Many planets in our solar system have magnetic fields of their own. For example, the magnetic field at the surface of Jupiter is about 4.3G. What role the magnetic field plays on the upper atmosphere depends on their field strength. For the planets without intrinsic magnetic fields, the magnetic fields could be induced at the interaction area between the stellar and planetary winds. However, the occurrence of strong magnetic fields occurring at 
the surfaces of planets can lead to significant changes in the upper atmospheres of planets. Trammell et al.(2011) have presented a 3D isothermal magnetohydrodynamic model based on the stellar wind model(Mestel 1968) although some physical details and the interaction between stellar and planetary winds are not considered into it. To include fully physical processes, for example, magnetic field and collision of winds, a powerful 3D MHD model is required. It is beyond the scope of this paper. However, the potential role of magnetic field can be discussed in the current 1D model. In the following discussion, we assume the planetary flows are spherically symmetric, namely, the magnetic field does not change the geometry of planetary wind within magnetosphere, but the boundary of magnetosphere constraints the range of planetary flows.

The most important effect of magnetic field on the hydrodynamic escape is whether the magnetic field can change the locations of sonic points for different species. If the boundary of magnetosphere is greater than that of sonic point, the planet can still emit a transonic wind. Otherwise, the planetary wind will be suppressed to subsonic flow, even quenched. Grie $\beta$ meier et al. (2004) have estimated the stand off distance of magnetosphere, $R_{s}$, by pressure balance and found that the stand-off distance of HD 209458b are about 2.6-3.8 $R_{p}$ which varied with different rotation velocities. Our results show that the locations of sonic points of $\mathrm{H}$ and $\mathrm{H}^{+}$are comparable with the distance of stand-off. As discussed previously(Section 3.1), the flow of atomic hydrogen could be transonic, but the escape of proton could be suppressed to subsonic flow. However, seen from Equations $(2),(3),(7)$ and (8), the drag force of $\mathrm{H}$ by $H^{+}$is negative if the velocity of $\mathrm{H}$ is higher than that of $H^{+}$. Thus the subsonic behavior of $H^{+}$could result in the decrease of the velocity of $\mathrm{H}$. In the process, charge exchange shifts momentum from one species with high velocity to the other species with low velocity. One possibility for the case is that both the flows become subsonic within magnetosphere and attain a new equilibrium status. As a consequence of the case, the mass loss rates of $\mathrm{H}$ and $\mathrm{H}^{+}$could decrease a factor of a few(Murray-Clay 
et al. 2009). In fact, the stand-off distance depends strongly on the strength of magnetic field, and the field strength could vary with the mass and evolution of planet(Reiners \& Christensen 2010; Scharf 2010). The capture to radio signal can answer what the intrinsic magnetic field is, however, no signal from the observations has been found so far (Lazio et al. 2010).

Note that the pressure of gas is omitted in the calculation of Grie $\beta$ meier et al. (2004) so that the stand-off distance could increase if the gas contributes a significant pressure fraction. Johansson et al. (2009) indicated the influence of expanding atmosphere on the stellar interaction. For unmagnetized exoplanets, the ram pressure of ionosphere can push the bow shock toward the host stars. The results hints that if the contribution of gas to the total pressure is included, the magnetopause of exoplanet could be outside of sonic points.

The ionization degree of hydrogen is not controlled explicitly by its velocity structure. Equation(1) shows clearly that the ionization of hydrogen is dominated by photoionizaiton. The magnetic fields have an indirectly effect on the ionization by changing the mass loss rates of planets. If the mass loss rates decrease a factor of a few, we can predict that there is a higher ionization degree in the atmosphere because the optical depth will also decrease.

For the exoplanet with an intrinsic dipole magnetic field, the ions near the equator could be inhibited by planetary magnetic field while the neutrals can escape freely from the atmosphere. At the portion of the planetary surface occupied by the closed magnetic fields hydrostatic equilibrium has been attained. Ions can not escape from the closed magnetic field although the neutrals can do so. Trammell et al.(2011) predicted a mass loss rate of $10^{9} \mathrm{~g} / \mathrm{s}$ when the magnetic field was considered into their model. For the details, reader may refer to the corresponding 2D or 3D models(Trammell et al.2011; Adams 2011). Our model should be suitable in the polar regions where the magnetic lines are open and almost aligned with the radial direction. Thus, the neutrals can escape from the whole surface of 
planet. But, the escaping surface of ions could decrease at least a factor of 2 (Yelle 2004). For the low ionization wind, the intrinsic dipole magnetic fields only confine a small part of the wind. For the high ionization wind in which ions are main composition, the intrinsic magnetic field can severely decrease the mass loss rate.

\section{Conclusions}

We have developed a multi-fluid model to describe the particle escape from the upper atmosphere of close-in planet. The continuity and momentum equations for each component were solved together with an energy equation. Detailed micro-physical process, for example, photoionization, recombination and charge exchange, have been included in our models. Based on Henyey method, the code can treat systems of ordinary differential equations with one, or more, critical points.

We calculated two samples of close-in planet, HD 209458b and HD 189733b. Our calculations show that the mass loss rates of two planets are of the order of magnitude of $10^{10}-10^{11}{\mathrm{~g} s^{-1}}$ which agree with the observed mass loss rates of $10^{10}-10^{11} \mathrm{~g} s^{-1}$. By detailed test and verification, we found that the most important physical process in the atmosphere of hot Jupiter is charge exchange which tightly couples atomic hydrogen with protons. Most of the hydrogen escaping from hot Jupiters is protons, especially in young star-planet system. Thus, the transit of Lyman- $\alpha$ in HD 189733b could be induced by other unknown physics processes.

In the assumption of single-fluid all species must remain collide frequently. Otherwise, the description of single-fluid can not be used. Our method can be further apply to other case, for example, the tenuous wind. It is found that decoupling may occur if the mass loss rates are lower than $10^{9} \mathrm{~g} / \mathrm{s}$. 
We also found that the assumption of energy-limit is not appropriate for ionized winds. Our model predicted a mass loss rate lower than that of energy-limit. By calculating the variations of mass loss rate with UV fluxes we fitted the mass loss rates of ionized wind as a function of $F_{U V}$.

\section{Acknowledgments}

This work was supported by National Natural Science Foundation of China(Nos.10803018)and Western Light Talent Culture Project of The Chinese Academy of Sciences(08AXB31001). I thank Ruth A Murray-Clay for helpful discussion in their single-fluid model. I also thank the referee for feedback that improved the paper. 


\section{REFERENCES}

Adams, F. C. 2011, arXiv:1101.4234

Bakos, G. A., Knutson, H., \& Pont, F., et al. 2006, ApJ, 650, 1160

Baraffe, I., Selsis, F., Chabrier, G., Barman, T. S., Allard, F., Hauschildt, P. H., \& Lammer, H. 2004, A\&A, 419, L13

Baraffe, I., Chabrier, G., Barman, T. S., Selsis, F., Allard, F., \& Hauschildt, P. H. 2005, A\&A, 436, L47

Ben-Jaffel, L., \& Hosseini, S. S. 2010, ApJ, 709, 1284

Bürgi, A. 1992, JGR, 97, A3, 3137

Davis, T. A., \& Wheatley, P. J. 2009, MNRAS, 396, 1012

Erkaev, N. V., Penz, T., Lammer, H., Lichtenegger, H. I. M., Biernat, H. K., Wucz, P., Grie $\beta$ Meier, J. M., \& Weiss, W. W. 2005, ApJs, 157, 396

Erkaev, N. V., Kulikov, Y. N., Lammer, H., Selsis, F., Langmayr, D., Jaritz, G. F., \& Biernat, H. K. 2007, A\&A, 472, 329

Ekenbäck, A., Holmström, M., Wucz, p., Griessmeier, J. M., Lammer, H., Selsis, F., \& Penz, T. 2010, ApJ, 709, 670

Garcia Munoz, A. 2007, Planet. Space Sci., 55, 1426

Geiss, J., Bürgi, A. 1986, A\&A, 159, 1

Grießmeier, J. M., Stadelmann, A., Penz, T., et al. 2004, A\&A, 425, 753

Guo, J. H. 2010, ApJ, 712, 1107 
Holmström, M., Ekenbäck, A., \& Selsis, F., et al. 2008, Nature, 451, 970

Hubbard, W. B., Hattori, M. F., \& Burrows, A., et al. 2007a, ApJ, 658, L59

Hubbard, W. B., Hattori, M. F., \& Burrows, A., et al. 2007b, Icarus, 187, 358

Ibgui, L., \& Burrows, A. 2009, ApJ, 700, 1921

Johansson, E. P. G., Bagdonat, T., \& Motschmann, U. 2009, A\&A, 496, 869

Koskinen, T. T., Yelle, R. V., Lavvas, P. L., \& lewis, N. K. 2010, ApJ, 723, 116 (K10)

Lammer, H., Selsis, F., Ribas, I., Guinan, E. F., Bauer, S. J., \& Weiss, W. W. 2003, ApJ, 598, L121

Lammer, H., Odert, P., Leitzinger, M., \& Weiss, W. W., et al. 2009, A\&A, 506, 399

Lazio, T., Joseph W., Clarke, Tracy E., et al. 2010, AJ, 140, 1995

Lecavelier des Etangs, A., Vidal-Madjar, A., McConnell, J. C., \& Hbrard, G. 2004, A\&A, 418, L1

Lecavelier Des Etangs, A., Ehrenreich, D., Vidal-Madjar, A., Ballester, G. E., Dsert, J.-M., Ferlet, R., Hbrard, G., Sing, D. K., Tchakoumegni, K.-O., \& Udry, S. 2010, A\&A, 514,72

Mestel, L. 1968, MNRAS, 138, 359

Murray-Clay, R. A., Chiang, E. I., \& Murray, N. 2009, ApJ, 693, 23

Nobili, L., \& Turolla, R. 1988, ApJ, 333, 248

Nordström, B., Mayor, M., \& Andersen, J., et al. 2004, A\&A, 418, 989

Penz,T., \& Micela, G. 2008, A\&A, 479, 579 
Penz,T., Erkaev, N. V., \& Kulikov, Y. N., et al. 2008, Planet. Space Sci., 56, 1260

Penz,T., Micela, G., \& Lammer, H. 2008, A\&A, 477, 309

Reiners, A., \& Chriestensen, U. R. 2010, A\&A, 522, A13

Sanz-Forcada, J., Ribas, I., Micela, G., Pollock, A. M. T., Garcia-Alvarez, D., Solano, E., \& Eiroa, C. 2010, A\&A, 511, L8

Scharf, C. A. 2010, ApJ, 722, 1547

Schunk, R. W. 1975, Planet. Space Sci., 23, 437

Shematovich, V. I. 2010, Sol. Syst. Res. 44, 96

Southworth, J. 2010, MNRAS, 408, 1689

Tian, F., Toon, O. B., Pavlov, A. A., \& De Sterck, H. 2005, ApJ, 621, 1049

Trammell, G, B., Arras, P., \& Li, Z. Y. 2011, ApJ, 728, 152

Vidal-Madjar, A., Lecavelier des Etangs, A., Dsert, J.-M., Ballester, G. E., Ferlet, R., Hbrard, G., \& Mayor, M. 2003, Nature, 422, 143 (VM03)

Vidal-Madjar, A., Dsert, J.-M., \& Lecavelier des Etangs, A., et al. 2004, ApJ, 604, L69

Winn, J. N., Holman, M. J., Henry, G. W., et al., 2007, AJ, 133, 1828

Watson, A. J., Donahue, T. M., \& Walker, J. C. G. 1981, Icarus, 48, 150

Yelle, R. 2004, Icarus, 170, 167

Yelle, R. 2006, Icarus, 183, 508 